\title{
A Novel Infection Protocol in Zebrafish Embryo to Assess Pseudomonas aeruginosa Virulence and Validate Efficacy of a Quorum Sensing Inhibitor In Vivo
}

\author{
Pauline Nogaret ${ }^{1,2}\left(\mathbb{D}\right.$, Fatima El Garah ${ }^{3}(\mathbb{D})$ and Anne-Béatrice Blanc-Potard 1,2,*(D) \\ 1 Laboratory of Pathogen-Host Interactions (LPHI), Université Montpellier, CEDEX 05, 34095 Montpellier, \\ France; pauline.nogaret@umontpellier.fr \\ 2 CNRS, UMR5235, CEDEX 05, 34095 Montpellier, France \\ 3 Laboratoire de Génie Chimique, Université de Toulouse, CNRS, INPT, UPS, 31432 Toulouse, France; \\ fatima.el-garah@univ-tlse3.fr \\ * Correspondence: anne.blanc-potard@univ-montp2.fr
}

Citation: Nogaret, P.; El Garah, F.; Blanc-Potard, A.-B. A Novel Infection Protocol in Zebrafish Embryo to Assess Pseudomonas aeruginosa Virulence and Validate Efficacy of a Quorum Sensing Inhibitor In Vivo. Pathogens 2021, 10, 401. https:// doi.org/10.3390/pathogens10040401

Academic Editor: Natalia Kirienko

Received: 10 March 2021

Accepted: 26 March 2021

Published: 29 March 2021

Publisher's Note: MDPI stays neutral with regard to jurisdictional claims in published maps and institutional affiliations.

Copyright: (c) 2021 by the authors. Licensee MDPI, Basel, Switzerland. This article is an open access article distributed under the terms and conditions of the Creative Commons Attribution (CC BY) license (https:// creativecommons.org/licenses/by/ $4.0 /)$.

\begin{abstract}
The opportunistic human pathogen Pseudomonas aeruginosa is responsible for a variety of acute infections and is a major cause of mortality in chronically infected cystic fibrosis patients. Due to increased resistance to antibiotics, new therapeutic strategies against $P$. aeruginosa are urgently needed. In this context, we aimed to develop a simple vertebrate animal model to rapidly assess in vivo drug efficacy against $P$. aeruginosa. Zebrafish are increasingly considered for modeling human infections caused by bacterial pathogens, which are commonly microinjected in embryos. In the present study, we established a novel protocol for zebrafish infection by $P$. aeruginosa based on bath immersion in 96-well plates of tail-injured embryos. The immersion method, followed by a 48-hour survey of embryo viability, was first validated to assess the virulence of P. aeruginosa wild-type PAO1 and a known attenuated mutant. We then validated its relevance for antipseudomonal drug testing by first using a clinically used antibiotic, ciprofloxacin. Secondly, we used a novel quorum sensing (QS) inhibitory molecule, $N$-(2-pyrimidyl)butanamide (C11), the activity of which had been validated in vitro but not previously tested in any animal model. A significant protective effect of C11 was observed on infected embryos, supporting the ability of C11 to attenuate in vivo $P$. aeruginosa pathogenicity. In conclusion, we present here a new and reliable method to compare the virulence of $P$. aeruginosa strains in vivo and to rapidly assess the efficacy of clinically relevant drugs against $P$. aeruginosa, including new antivirulence compounds.
\end{abstract}

Keywords: Pseudomonas aeruginosa; zebrafish; quorum sensing

\section{Introduction}

The environmental bacterium and opportunistic human pathogen Pseudomonas aeruginosa is responsible for a variety of acute infections and is a major cause of mortality in chronically infected cystic fibrosis (CF) patients. Due to increased resistance to antibiotics, $P$. aeruginosa has been listed by the World Health Organization (WHO) among pathogenic bacteria for which new antibiotics or alternative therapeutic strategies are urgently needed [1]. Antivirulence strategies have emerged as attractive novel therapeutic approaches that would apply less selective pressure to develop resistance and better preserve microbiota than traditional antimicrobial therapy [2,3].

Quorum sensing (QS) allows bacteria to communicate with one another by responding to the population-dependent concentration of small molecules known as autoinducers [4]. $P$. aeruginosa secretes two main classes of autoinducer: acyl-homoserine lactones (HSLs) and several alkylquinolones (AQs), the major one being 2-heptyl-3-hydroxy-4-quinolone (PQS). P. aeruginosa harbors three main QS systems (las, rhl, and pqs) that control potent virulence factors [5] and are essential for P. aeruginosa pathogenesis, as demonstrated by 
a study in which P. aeruginosa mutants that lacked QS genes caused less lung pathology during acute infection in mice [6]. In addition, sputum cultures from CF patients infected with chronic P. aeruginosa contain significant amounts of HSLs and PQS [7]. Thus, QS systems of $P$. aeruginosa have been proposed as potential antivirulence targets [8,9]. In this context, a promising strategy for curtailing $P$. aeruginosa virulence based on a structural analog of $\mathrm{C}_{4}$-HSL autoinducer has been identified $[10,11]$. This compound, named C11 (N(2-pyrimidyl)butanamide), downregulates the las and $r h l$ QS systems and notably reduces biofilm formation, without affecting P. aeruginosa planktonic growth [10]. Moreover, a synergistic antibiofilm activity was found between $\mathrm{C} 11$ and the clinically used antibiotics ciprofloxacin, tobramycin, and colistin [10]. Thus, C11 appears as a promising antivirulence molecule that is suitable for in vivo studies because it is stable, not cytotoxic to human cells, and synthetically accessible.

In the context of validation of novel compounds against $P$. aeruginosa, the development of low-cost and rapid animal models is important to assess in vivo efficacy. The zebrafish (Danio rerio) has been now widely used as a model for studying host-pathogen interactions [12,13] and is increasingly considered for modeling human infections, including lung infections caused by bacterial pathogens $[14,15]$. The zebrafish model has a number of advantages over mammalians models of infection in terms of methodological, financial, and ethical issues. Zebrafish are vertebrates, which are genetically and physically closer to humans than invertebrate models. In embryos, only the innate immune system is functional [16], and the optical transparency of the embryonic stages allows the analysis of bacterial infections in real time using fluorescent microorganisms. In addition to its significance as an infection model, the zebrafish embryo is also suitable for in vivo chemical screening $[17,18]$, with the advantage that permeability of the larvae allows the entry of small compounds added directly to the fish water. This model, which also allows drug toxicity to be addressed [19], has been successfully used for drug testing in the context of cancer and infectious diseases $[20,21]$.

The zebrafish embryo model has been used to follow P. aeruginosa infections and assess $P$. aeruginosa virulence [22-25]. P. aeruginosa infections are usually established by microinjecting the bacteria into the bloodstream (injection in caudal vein or duct of Cuvier) of 1 or 2 days post-fertilization (dpf) old embryos. This induces an acute infection and mortality of embryos when the amount of bacteria injected exceeds the phagocytic capacity of the embryo. P. aeruginosa mutants deficient in QS (lasR and mvfR) are attenuated in zebrafish embryos infected at $2 \mathrm{dpf}$ [23], supporting that the zebrafish embryo is a suitable infection model to test the efficacy of specific inhibitors targeting QS.

In the present study, we first aimed to develop a simple and reliable model for $P$. aeruginosa infection in zebrafish embryos based on bath immersion, thus avoiding the time-consuming microinjection step. This new protocol, which is carried out with injured embryos, was first validated to assess $P$. aeruginosa virulence. We next validated its reliability for drug testing using a clinically used antibiotic and the promising C11 QS inhibitory molecule.

\section{Results}

\subsection{A Bath Infection Model with Injured Zebrafish Embryos Allows the Evaluation of P. aeruginosa Virulence}

$P$. aeruginosa infection in zebrafish embryos is usually established through microinjection of the bacteria into the embryo, which requires specific expertise and is time-consuming. Our first goal was to set up a reproducible infection assay based on the immersion of embryos with bacteria to bypass the microinjection step. We also aimed to set up a test in 96-well plates to minimize the bath volume in further drug assays. A previous study reported mortality of embryos after bath immersion with PAO1 strain [26], which is, however, not supported by other reports [23,27]. Bath immersion was first performed on healthy embryos at 2 days post-fertilization (dpf) [28], a developmental stage where the mouth is not yet open. We used different bacterial concentrations of a PAO1 strain with two embryos per well in 96-well plates. Viability of embryos was followed for 24 hours but no mortality 
was observed (Figure S1). The same experiment was then carried out with embryos injured in the tail fin (Figure 1A), because such injury has been shown to provide a portal of entry for bacteria [29]. In contrast to healthy embryos, mortality of injured embryos was observed after 18 hours post-infection (hpi) (Figure 1B), in a bacterial dose-dependent manner (Figure 1C). Imaging embryos shortly after infection revealed the presence of bacteria in the tail 1.5 hours post-infection, which can persist in some live embryos 24 hours after bathing (Figure 1D). Thus, the bath infection model with embryos wounded in the tail appeared a suitable novel mode of infection by P. aeruginosa. A deeper microscopy analysis at additional time points will be required for a better understanding of the pathogenesis process. While the image in Figure 1D for time 1.5 hours is representative of the majority of embryos, the image of an alive embryo at time 24 hours is not representative of the diversity of patterns (some embryos cleared the infection and some embryos harbored bacteria that had migrated away from the injury site). We also noticed some variability in the embryo susceptibility to infection, which may depend on various factors, including the age of adult fish. In further experiments, we adjusted the bacterial concentration to achieve a mortality of 60 to $80 \%$ of embryos upon infection with PAO1.

A

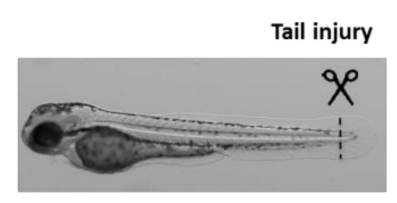

B

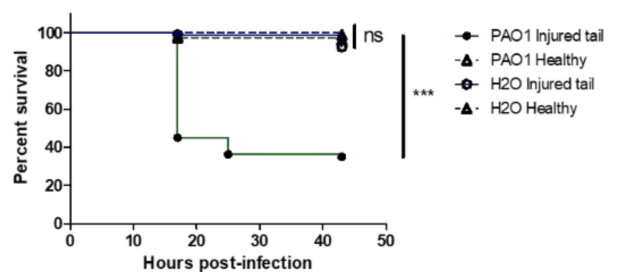

C

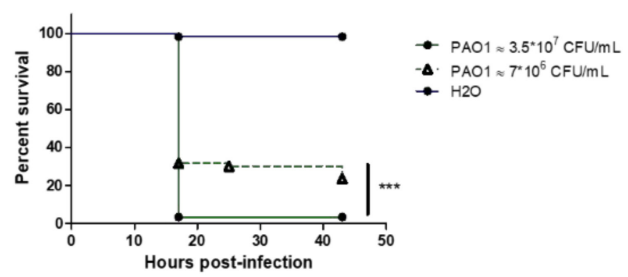

D

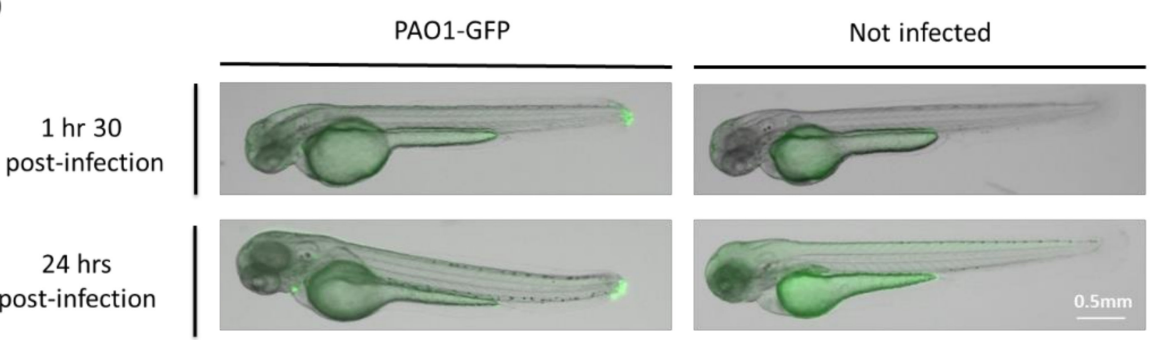

Figure 1. Development of bath infection model with injured zebrafish embryos to assess P. aeruginosa virulence. (A) Larvae were injured in the tail fin at 48 hours post-fertilization (hpf). (B) Survival curves (Kaplan-Meier representation) of healthy or injured embryos (GAB line) immersed at $48 \mathrm{hpf}$ with PAO1 wild-type strain at approximately $2^{*} 10^{7} \mathrm{CFU} / \mathrm{mL}$ grown in exponential phase or "fish water" (negative control). (C) Survival curves (Kaplan-Meier representation) of injured embryos (GAB line) immersed at $48 \mathrm{hpf}$ with PAO1 strain at two different concentrations $\left(3.5 \times 10^{7}\right.$ or $\left.7 \times 10^{6} \mathrm{CFU} / \mathrm{mL}\right)$ or fish water (negative control). Results are expressed as the percentage of surviving embryos. Pools of three biologically independent replicates are shown for each survival curve (in each experiment 20 embryos were used per strain). Significant difference at ${ }^{* *} p<0.01,{ }^{* * *} p<0.001$ or no significant difference: ns. (D) In vivo imaging of injured embryos immersed with fluorescent P. aeruginosa PAO1-GFP for 1.5 or 24 hours (left panels). Images represent DIC channel merge to green fluorescence protein (GFP) channel. Scale bar: $0.5 \mathrm{~mm}$. Images of injured embryos immersed with N-phenylthiourea (PTU) without bacteria (right panels) are shown as control. Images are a representative result of three embryos in each condition.

OprF is a major outer membrane porin involved in maintenance of cell structure, outer membrane permeability, environmental sensing, adhesion, biofilm formation, and virulence [30,31]. To validate the relevance of the new infection model for the assessment of $P$. aeruginosa virulence, we performed a test with wild-type PAO1 and an oprF mutant strain, which was previously shown to be attenuated in zebrafish embryos when bacteria are microinjected in the caudal vein [32]. The attenuation profile of the oprF mutant in comparison with wild-type PAO1 is similar upon classical microinjection of the bacteria 
in the caudal vein (Figure 2A) or upon bath infection of injured embryos (Figure 2B), supporting the reliability of the bath model in assessing P. aeruginosa virulence.

A

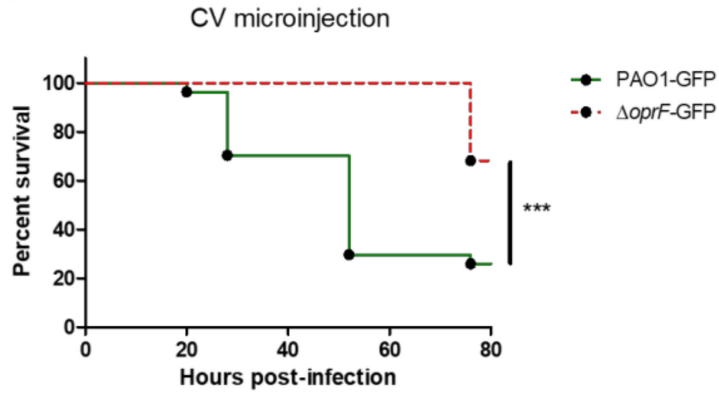

B

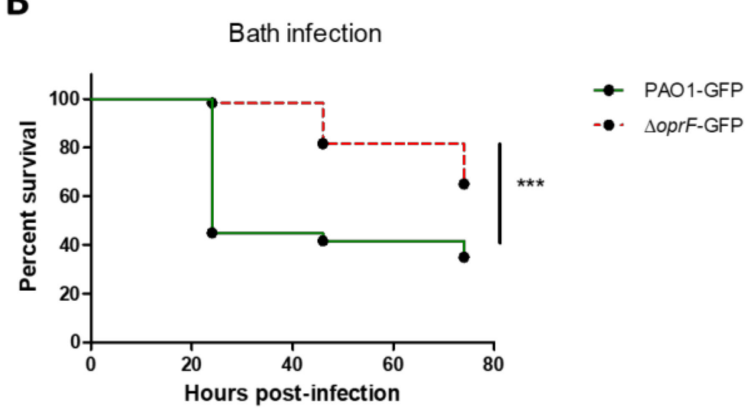

Figure 2. Validation of bath infection model using an attenuated strain of P. aeruginosa. (A) Kaplan-Meier representation of survival after microinjection of approximately 3500 CFUs of wild-type or $\triangle$ oprF GFP-expressing P. aeruginosa into the caudal vein (CV) of embryos. Pools of three biologically independent replicates are shown (in each experiment 20 embryos were used per strain). (B) Kaplan-Meier representation of survival after bath infection of injured embryos with wild-type or $\Delta o p r F$ GFP-expressing P. aeruginosa at approximately $10^{8} \mathrm{CFU} / \mathrm{mL}$. Pools of three biologically independent replicates are shown for each survival curve (in each experiment 20 embryos were used per strain).

\subsection{Validation of the Bath Infection Model to Test Efficacy of a Known Antibiotic}

We next addressed the relevance of the bath infection model to test drug efficacy using ciprofloxacin, a known antipseudomonal antibiotic. Ciprofloxacin added directly in the bath at $50 \mu \mathrm{g} / \mathrm{mL}$ was previously shown to rescue $75 \%$ of embryos from lethal infection following microinjection at 50 hours post-fertilization (hpf) with P. aeruginosa PA14 strain [23]. In our model, ciprofloxacin was added 2 hours after injury and bacterial immersion, to allow the wound closing and avoid a direct killing of extracellular bacteria in the bath. Ciprofloxacin at $50 \mu \mathrm{g} / \mathrm{mL}$ had a clear and significant protective effect compared to nontreated embryos (Figure $3 \mathrm{~A}$ ). A significant protective effect, of lower amplitude, was also shown at a lower dose of $1 \mu \mathrm{g} / \mathrm{mL}$ (Figure 3B), which corresponds to 10-fold minimum inhibitory concentration (MIC) [33]. Bath infection of injured embryos with P. aeruginosa is thus suitable to evaluate the efficacy of antibacterial drugs.
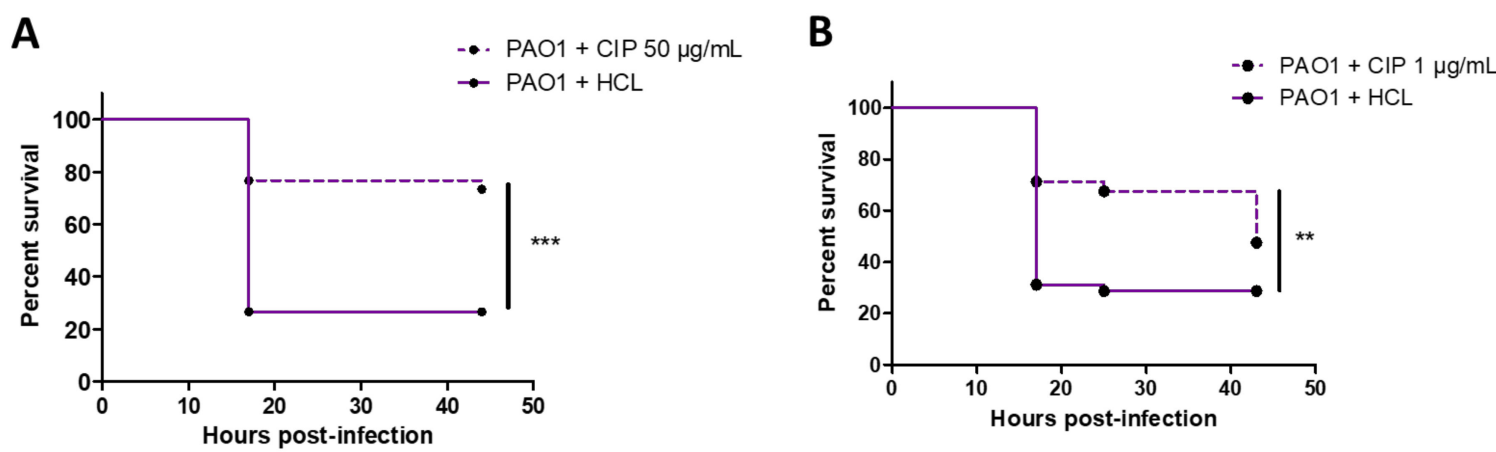

Figure 3. Validation of bath infection model with a known antibiotic. (A) Kaplan-Meier representation of the survival of injured zebrafish embryos bath infected with PAO1 strain at approximately $10^{8} \mathrm{CFU} / \mathrm{mL}$ and treated with ciprofloxacin $50 \mu \mathrm{g} / \mathrm{mL}$ or hydrochloric acid $0.2 \mathrm{mM}$ (negative control) 2 hours after infection. (B) Kaplan-Meier representation of the survival of injured zebrafish embryos bath infected with PAO1 strain at approximately $5 \times 10^{7} \mathrm{CFU} / \mathrm{mL}$ and treated with ciprofloxacin $1 \mu \mathrm{g} / \mathrm{mL}$ or hydrochloric acid $0.5 \mathrm{mM}$ (negative control) 2 hours after infection. Pools of three biologically independent replicates (with 20 embryos) are shown for each survival curve. ${ }^{* * *} p<0.001 ;{ }^{* *} p<0.01$. 


\subsection{Test of Antivirulence Properties of C11, a Novel Anti-Pseudomonas Molecule Targeting QS}

We next evaluated the ability of the protocol of bath infection of injured embryos to test an antivirulence compound in vivo. C11, an antagonistic analog of $\mathrm{C}_{4}-\mathrm{HSL}$, is a novel molecule targeting P. aeruginosa QS [10] that had not previously been evaluated in an animal model. The effect of C11, dissolved in dimethylsulfoxide (DMSO), was tested at different concentrations $(10,25$, and $50 \mu \mathrm{M})$ on injured embryos in bath immersion with bacterial inoculum at about $10^{7} \mathrm{CFU} / \mathrm{mL}$. C11 has been previously shown to lack classical antibacterial activity and was, therefore, added simultaneously with the bacterial cells following the protocol schematized in Figure 4A. In control embryos immersed with PAO1, DMSO was added to reflect the amount of DMSO in C11-treated embryos $(0.05$, 0.13 , or $0.25 \%$ ). The $\mathrm{C} 11$ compound exhibits a protective effect since a significant reduction in embryo mortality was observed at 25 and $50 \mu \mathrm{M}$ (Figure 4B). This protective effect was not observed at $10 \mu \mathrm{M}$ (Figure $4 \mathrm{~B}$ ), thus demonstrating a dose-dependent effect. After this first round of experiments with C11 dissolved in DMSO, we also checked its efficiency upon dilution in water, as previously used [10]. C11 diluted in water at $25 \mu \mathrm{M}$ was significantly protective (Figure S2), to a similar extent to the effect observed upon dilution in DMSO, thus supporting that DMSO does not contribute to C11 activity in vivo. Importantly, the addition of $25 \mu \mathrm{M} \mathrm{C} 11$ (dissolved in DMSO or $\mathrm{H}_{2} \mathrm{O}$ ) was not associated with toxicity for embryos, as shown by the lack of mortality upon addition of the sole molecule (Figures $4 \mathrm{C}$ and S2).

A

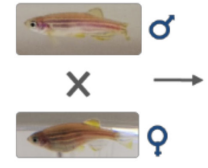

Crossing zebrafish

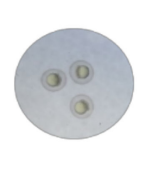

Egg collection

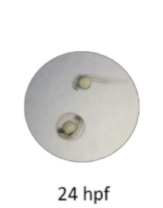

Egg dechorionation

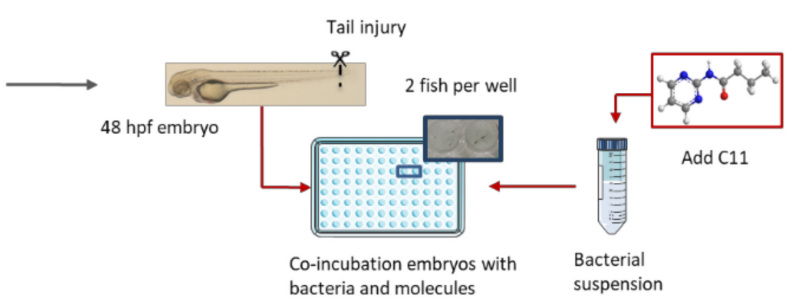

bacteria and molecules
B
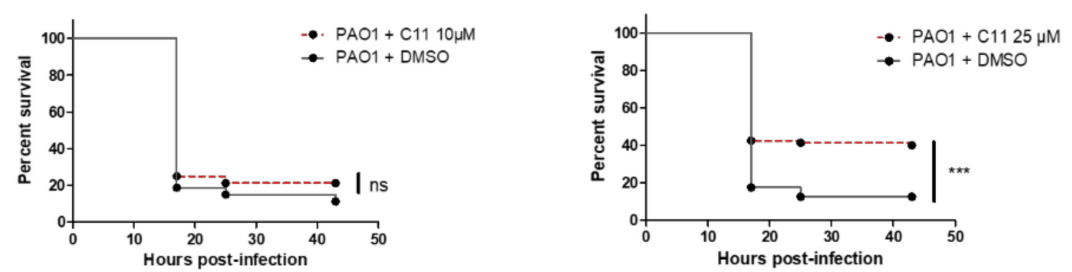

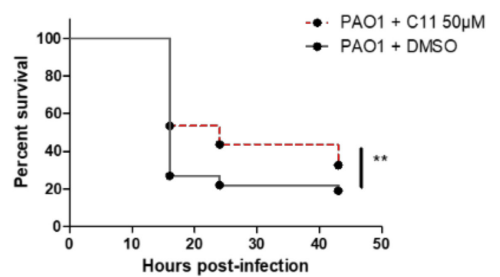

C

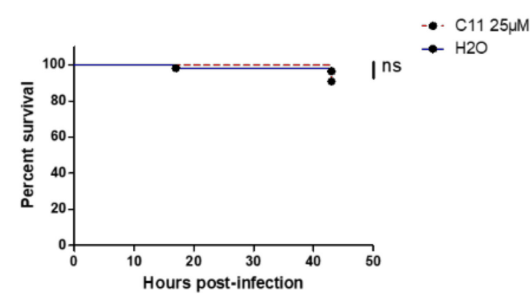

Figure 4. Use of bath infection model to validate novel anti-Pseudomonas molecules such as C11 molecule (targeting quorum sensing). (A) Schematic protocol for testing antivirulence molecules in embryos bath infected with P. aeruginosa. Antivirulence molecules, which do not reduce bacterial viability outside the host, are added together with bacteria. (B) The antivirulence efficacy of C11 (dissolved in DMSO) was tested with embryos injured in the tail fin and bath infected with PAO1 suspension at approximately $10^{7} \mathrm{CFU} / \mathrm{mL}$ in presence of $\mathrm{C} 11$ at 10, 25, or $50 \mu \mathrm{M}$. Injured embryos in the control group were treated with PAO1 suspension in presence of DMSO at $0.05,0.13$, or $0.25 \%$ (reflecting the amount of DMSO in the C11-treated groups). A significant difference $\left.{ }^{* *} p<0.05,{ }^{* * *} p<0.001\right)$ is found in the survival curve of C11-treated embryos compared to non-treated embryos at 25 or $50 \mu \mathrm{M}$. (C) The toxicity of C11 at $25 \mu \mathrm{M}$ was monitored after immersion of embryos injured in the tail fin. For all experiments, the embryo survival was monitored for 45 hours and survival curves were represented with a Kaplan-Meier representation. Graphs represent the pool of three independent experiments $(n=60$ larvae in total per condition). 


\section{Discussion}

The use of the zebrafish embryo as in vivo model for human diseases offers a great opportunity to conduct chemical screens for small molecules in a whole-organism model [34]. The relevance of this model for testing novel therapeutics against $P$. aeruginos $a$ is supported by a recent study using phages [35]. In the present work, we used zebrafish larvae infected by $P$. aeruginosa to test antivirulence molecules for the first time. We firstly developed a novel protocol for infection of zebrafish embryos to assess $P$. aeruginosa virulence and secondly demonstrated its relevance in evaluating the efficacy of antipseudomonal molecules.

$P$. aeruginosa infection in zebrafish embryos is most often established through microinjection of bacterial cells into the embryo. In the context of in vivo drug testing, our aim was first to develop an immersion protocol with P. aeruginosa to avoid the microinjection step. A lethal infection after bath immersion of $3 \mathrm{dpf}$ healthy embryos with $P$. aeruginosa was previously reported [26]. However, an associated proteomic analysis indicated induction of hypoxia response in zebrafish embryos exposed to the immersion method, suggesting that healthy larvae suffered from a lack of oxygen when exposed to $P$. aeruginosa by static immersion. Moreover, the host response was also likely corresponding to a response against bacteria that are outside the fish or in contact with its skin [26]. In the present study, we did not observe lethality when healthy embryos at $2 \mathrm{dpf}$ (a developmental stage where the mouth is not yet open) were immersed with PAO1 bacteria, which is in agreement with other reports $[23,27]$. However, we observed bacterial-dose-dependent mortality when 2 dpf embryos were injured in the tail fin before static immersion with P. aeruginosa. Fin amputation in embryos has been widely used as a model of "sterile" wounding injury and inflammation assay [36]. The inflammatory response with the arrival of neutrophils and macrophages after an injury has been well documented, and the embryonic zebrafish fin is also highly regenerative (the tail has been reported to heal within 1 hour after the cut) [37]. Moreover, the use of tail-injured embryos has been shown to increase the susceptibility to infection when compared to $4 \mathrm{dpf}$ healthy larvae by providing an alternative entry gate for the bacterium Aeromonas hydrophila [29]. We visualized fluorescent $P$. aeruginosa bacteria within embryos shortly after immersion, which can persist 24 hours after injury in surviving embryos. Thus, bath immersion of tail-injured embryos appears to be a suitable novel protocol to infect zebrafish embryos with P. aeruginosa. P. aeruginosa OprF was previously reported to play a role in resistance to macrophage clearance during acute infection in zebrafish embryos [32]. To ascertain the pertinence of the new infection mode, we have shown that an oprF mutant strain, which is attenuated upon microinjection [32], is similarly attenuated using bath immersion of tail-injured embryos. Further studies, implying deeper microscopy analysis of wild-type and mutant strains, as well as monitoring innate immune response, will be required to decipher the pathogenesis mechanism associated with this novel infection route.

The relevance of tail-injured embryos in assessing the efficacy of drugs against $P$. aeruginosa was first validated with the clinically used antibiotic ciprofloxacin. Protection of embryos from mortality caused by infection was observed at $50 \mu \mathrm{g} / \mathrm{mL}$, a concentration also previously found to be efficient in protecting embryos microinjected with P. aeruginosa [23]. Moreover, we showed that a dose as low as $1 \mu \mathrm{g} / \mathrm{mL}$ was sufficient to significantly protect embryos. After validation of the method with a known antibiotic, we tested a structural analog of $\mathrm{C}_{4}-\mathrm{HSL}$, the C11 molecule. C11 is a novel inhibitor of P. aeruginosa QS [10], and its activity in an animal model was evaluated for the first time in the present study. The addition of $\mathrm{C} 11$ reduced embryo mortality, with a significant protective effect at 50 and $25 \mu \mathrm{M}$. Thus, our in vivo results confirmed the anti-infective potential of this novel anti-QS compound, without exhibiting any toxicity. Further studies will be required to better understand the mechanism by which C11 increases the defensive ability of zebrafish embryos against $P$. aeruginosa in vivo. In vitro, $\mathrm{C} 11$ was shown to inhibit $P$. aeruginosa biofilm formation and reduce the expression of QS regulatory genes and QS-regulated genes [10]. Because P. aeruginosa causes an acute infection in the zebrafish embryo model, the reduced embryo mortality observed upon C11 addition in vivo is likely 
due to its ability to downregulate QS gene expression, which in turn impairs virulence, rather than a proper anti-biofilm effect. In addition or alternatively, we cannot exclude that C11 may also promote immunomodulatory mechanisms in vivo and stimulate a protective innate immune response.

To summarize, we have validated the use of static immersion of injured embryos for the assessment of $P$. aeruginosa virulence and the evaluation of anti-Pseudomonas molecules for the first time. The main advantage of this method is its rapidity and feasibility, making it suitable for the in vivo screening of novel anti-P. aeruginosa molecules. This method, which has confirmed the therapeutic potential of the C11 anti-QS compound, should thus be suitable to validate in vivo other molecules targeting QS, as well as molecules targeting other virulence factors important for P. aeruginosa pathogenesis in zebrafish embryos. In the current model, P. aeruginosa causes an acute infection, and it would be of importance to identify conditions for a more persistent infection to also assess the efficiency of QS inhibitors in such conditions.

\section{Materials and Methods}

\subsection{Bacterial Strains and Growth Conditions}

We used P. aeruginosa PAO1 strain or PAO1 (H103) constitutively expressing green fluorescence protein (GFPmut2) [38] to visualize bacteria in zebrafish embryos. GFPexpressing oprF mutant (H636) was described previously [32]. Bacteria were grown at $37^{\circ} \mathrm{C}$ in Luria broth (LB). Carbenicillin $(150 \mu \mathrm{g} / \mathrm{mL})$ was added for strains expressing GFP. Bacterial growth was monitored in 96-well plates using a Spark 20M (Tecan, Trading AG, Männedorf, Switzerland) microplate reader.

\subsection{Chemicals}

N-(2-Pyrimidyl)butanamide (MW 165.19), named C11, was synthesized as described [10,39] and dissolved at $19 \mathrm{mM}$ in DMSO or $\mathrm{H}_{2} \mathrm{O}$. For bath infection, the stock solution was first diluted to $1.9 \mathrm{mM}$ by adding phosphate-buffered saline (PBS) drop by drop and then diluted at the indicated concentration $(10,25$, or $50 \mu \mathrm{M})$.

Ciprofloxacin (Sigma-Aldrich, St-Louis, MO, USA, MW 331.35) was dissolved at $25 \mathrm{mg} / \mathrm{mL}$ in $0.1 \mathrm{M}$ hydrochloric acid.

\subsection{Bath Infection of Danio rerio Embryos with P. aeruginosa}

Bacterial strains, freshly streaked out from glycerol stock, were grown overnight at $37{ }^{\circ} \mathrm{C}$ in LB medium (with antibiotic when required). Cultures were diluted 1:20 in LB medium and grown until $\mathrm{OD}_{600}=0.9$. Bacteria were centrifuged at $4000 \mathrm{rpm}$ for $10 \mathrm{~min}$ and resuspended at the desired concentration in fish water composed of $60 \mu \mathrm{g} / \mathrm{mL}$ sea salt (Instant Ocean, Blacksburg, VA, USA) in distilled water with $4.10^{-4} \mathrm{~N} \mathrm{NaOH}$ or Nphenylthiourea (PTU) (for experiments with microscopic analysis). The number of bacteria in the inoculum was determined by subsequent plating onto LB agar after dilution into PBS.

Experiments were performed using the $A B$ zebrafish line or the $G A B$ zebrafish line and maintained at $28{ }^{\circ} \mathrm{C}$ under standard conditions in fish water [25]. Bath immersion infections were done at $48 \mathrm{hpf}$ on embryos dechorionated at $30 \mathrm{hpf}$. To injure the tail fin before infection, embryos were placed in a Petri dish and anesthetized with $40 \mu \mathrm{g} / \mathrm{mL}$ tricaine, and a small transection of the tail was performed with a 26-gauge needle under a stereomicroscope (Motic, Barcelona, Spain).

For initial experiments on healthy embryos, groups of 20 larvae were distributed into 6-well plates (Falcon) containing $4.0 \mathrm{~mL}$ of fish water or bacterial suspension. For experiments on injured embryos, groups of 20 injured embryos were first immersed in a Petri dish containing bacterial suspension (or fish water as control) immediately after the transection of the tail and were then distributed into 96-well plates (Falcon ${ }^{\mathrm{TM}}$, Glendale, AZ, USA) with two fish per well containing $200 \mu \mathrm{L}$ of bacteria suspension (or fish water). The plates were incubated at $28{ }^{\circ} \mathrm{C}$ (bacteria are kept throughout the experiment in fish 
water, which does not support P. aeruginosa growth). For survival kinetics after infection, the number of dead embryos was determined visually based on the absence of heartbeat.

\subsection{Fluorescence Microscopic Analysis of Zebrafish Embryos}

Embryos were maintained in fish water containing PTU 1X added at $24 \mathrm{hpf}$ to prevent pigment formation. Groups of five injured embryos were distributed into 6-well plates containing $4.0 \mathrm{~mL}$ of GFP-expressing bacteria suspension in PTU. After 1.5 or 24 hours of bath immersion, embryos were washed four times in PTU and kept for 50 min in PTU 1X. For live imaging, embryos from bath infection were placed in a glass-bottom Petri dish for inverted microscopy containing tricaine $(250 \mu \mathrm{g} / \mathrm{mL})$ in PTU. Direct visualization was performed using an Olympus MVX10 epifluorescent microscope equipped with a digital color camera (Olympus XC50). Fluorescence and bright-field images were acquired and processed with CellSens (Olympus) with GFP filter and assembled using ImageJ software to adjust levels and brightness of fluorescence.

\subsection{Infection of Danio rerio Embryos by Microinjection in the Caudal Vein}

Experiments were performed using the $\mathrm{AB}$ zebrafish and maintained under standard conditions [25]. Bacterial strains, which were freshly streaked out from glycerol stocks, were grown in LB medium to mid-log phase $\left(\mathrm{OD}_{600}=0.7-0.8\right)$, recovered by centrifugation, and washed twice in PBS. Suspensions were homogenized through a 26-gauge needle and resuspended in PBS at about $10^{9}$ bacteria/mL added with $10 \%$ phenol red to aid visualization of the injection process. Infection was carried by the direct microinjection of $2 \mathrm{~nL}$ of bacterial suspensions into the caudal vein of $50 \mathrm{hpf}$ embryos, previously dechorionated and anesthetized with $0.02 \%$ tricaine. For survival kinetics after infection, the number of dead embryos was determined visually based on the absence of heartbeat.

\subsection{Treatment of Bath Infected Embryos with Ciprofloxacin or C11}

All experiments were conducted on groups of 20 injured embryos in 96-well plates (two embryos per well) containing $200 \mu \mathrm{L}$ of fish water supplemented with bacterial suspension and molecule or solvent control. Toxicity was addressed with embryos treated only with the molecule but not bacteria.

Ciprofloxacin was added after 2 hours of incubation to each well containing injured embryos to reach a final concentration of 50 or $1 \mu \mathrm{g} / \mathrm{mL}$. Negative control was done with $\mathrm{HCl}(0.2$ and $0.5 \mathrm{mM}$, respectively).

Bacterial suspension was added drop by drop to $\mathrm{C} 11$ to reach a final concentration of 10,25 , or $50 \mu \mathrm{M}$ before immersion of injured embryos. For non-treated controls, bacterial suspension was added to adjusted volumes of DMSO to reflect the DMSO amount present in treated samples.

\subsection{Statistical Analysis}

All statistical analyses were performed using GraphPad Prism 5 (Graphpad Software, San Diego, CA, USA). To compare the distribution of two groups, the Kruskal-Wallis test was performed. Comparisons between survival curves were performed using the log-rank test. In figures, ${ }^{*}$ means $p$-value $<0.05,{ }^{* *}<0.01$, and ${ }^{* * *}<0.001$.

\subsection{Ethics Statement}

All animal experiments described in the present study were conducted at the University of Montpellier according to European Union guidelines for handling of laboratory animals (http:/ / ec.europa.eu/environment/chemicals/lab_animals/home_en.htm (accessed on 15 March 2021)) and were approved by the Direction Sanitaire et Vétérinaire de l'Hérault and Comité d'Ethique pour l'Expérimentation Animale under reference CEEALR- B4-172-37 and APAFIS\#5737-2016061511212601 v3. The breeding of adult fish adhered to the international guidelines specified by the EU Animal Protection Directive 2010/63/EU, and adult zebrafish were not sacrificed for this study. All experiments were performed 
before the embryos' free-feeding stage and did not fall under animal experimentation law according to the EU Animal Protection Directive 2010/63/EU. For survival curves, cardiac rhythm was used as a clinical criterium. Embryos were euthanized using the anesthetic tricaine up to a lethal dose $(500 \mathrm{mg} / \mathrm{mL})$ before bleach treatment.

Supplementary Materials: The following are available online at https://www.mdpi.com/article/10 .3390/pathogens10040401/s1, Figure S1: Immersion of healthy embryos with P. aeruginosa. Figure S2: Efficiency and toxicity of C11 molecule dissolved in water.

Author Contributions: Conceptualization, P.N. and A.-B.B.-P.; methodology, P.N.; validation, P.N. and A.-B.B.-P.; formal analysis, P.N., F.E.G., and A.-B.B.-P.; investigation, P.N. and A.-B.B.-P.; resources, F.E.G. and A.-B.B.-P.; writing-original draft preparation, A.-B.B.-P.; writing-review and editing, P.N., F.E.G., and A.-B.B.-P.; visualization, P.N. and A.-B.B.-P.; supervision, A.-B.B.-P.; project administration, A.-B.B.-P.; funding acquisition, F.E.G. and A.-B.B.-P. All authors have read and agreed to the published version of the manuscript.

Funding: This research was funded by Agence Nationale de la Recherche (ANR-17-CE18-0011-02) and Vaincre La Mucoviscidose and Association Gregory Lemarchal (RF20190502411). P.N. is supported by Agence Nationale de la Recherche (ANR-17-CE18-0011-02) and Vaincre La Mucoviscidose and Association Gregory Lemarchal (RF20190502411).

Institutional Review Board Statement: The study was conducted according to the guidelines of the Declaration of Helsinki and approved by Direction Sanitaire et Vétérinaire de l'Hérault and Comité d'Ethique pour l'Expérimentation Animale under reference CEEA-LR- B4-172-37 and APAFIS\#5737$2016061511212601 \mathrm{v} 3$.

Informed Consent Statement: Not applicable.

Acknowledgments: We thank Christine Roques (Toulouse University) for constructive discussion, Malika Moussouni for help with the microinjection experiment, and Cathy Gonzalez for fish facility maintenance.

Conflicts of Interest: The authors declare no conflict of interest.

\section{References}

1. Tacconelli, E.; Carrara, E.; Savoldi, A.; Harbarth, S.; Mendelson, M.; Monnet, D.L.; Pulcini, C.; Kahlmeter, G.; Kluytmans, J.; Carmeli, Y.; et al. Discovery, research, and development of new antibiotics: The WHO priority list of antibiotic-resistant bacteria and tuberculosis. Lancet Infect. Dis. 2018, 18, 318-327. [CrossRef]

2. Heras, B.; Scanlon, M.J.; Martin, J.L. Targeting virulence not viability in the search for future antibacterials. Br. J. Clin. Pharmacol. 2015, 79, 208-215. [CrossRef]

3. Mühlen, S.; Dersch, P. Anti-virulence Strategies to Target Bacterial Infections. In How to Overcome the Antibiotic Crisis; Current Topics in Microbiology and Immunology; Springer: Cham, Switzeland, 2015; Volume 398, pp. 147-183. [CrossRef]

4. Papenfort, K.; Bassler, B.L. Quorum sensing signal-response systems in Gram-negative bacteria. Nat. Rev. Genet. 2016, 14, 576-588. [CrossRef]

5. Jimenez, P.N.; Koch, G.; Thompson, A.; Xavier, K.B.; Cool, R.H.; Quax, W.J. The Multiple Signaling Systems Regulating Virulence in Pseudomonas aeruginosa. Microbiol. Mol. Biol. Rev. 2012, 76, 46-65. [CrossRef]

6. Pearson, J.P.; Feldman, M.; Iglewski, B.H.; Prince, A. Pseudomonas aeruginosa Cell-to-Cell Signaling Is Required for Virulence in a Model of Acute Pulmonary Infection. Infect. Immun. 2000, 68, 4331-4334. [CrossRef]

7. Collier, D.N.; Anderson, L.; McKnight, S.L.; Noah, T.L.; Knowles, M.; Boucher, R.; Schwab, U.; Gilligan, P.; Pesci, E.C. A bacterial cell to cell signal in the lungs of cystic fibrosis patients. FEMS Microbiol. Lett. 2002, 215, 41-46. [CrossRef]

8. Shaw, E.; Wuest, W.M. Virulence attenuating combination therapy: A potential multi-target synergy approach to treat Pseudomonas aeruginosa infections in cystic fibrosis patients. Rsc. Med. Chem. 2020, 11, 358-369. [CrossRef]

9. Smith, R.S.; Iglewski, B.H. Pseudomonas aeruginosa quorum sensing as a potential antimicrobial target. J. Clin. Investig. 2003, 112, 1460-1465. [CrossRef]

10. Furiga, A.; Lajoie, B.; El Hage, S.; Baziard, G.; Roques, C. Impairment of Pseudomonas aeruginosa Biofilm Resistance to Antibiotics by Combining the Drugs with a New Quorum-Sensing Inhibitor. Antimicrob. Agents Chemother. 2016, 60, 1676-1686. [CrossRef]

11. Khalilzadeh, P.; Lajoie, B.; El Hage, S.; Furiga, A.; Baziard, G.; Berge, M.; Roques, C. Growth inhibition of adherent Pseudomonas aeruginosa by an N-butanoyl-l-homoserine lactone analog. Can. J. Microbiol. 2010, 56, 317-325. [CrossRef]

12. Torraca, V.; Mostowy, S. Zebrafish Infection: From Pathogenesis to Cell Biology. Trends Cell Biol. 2018, 28, 143-156. [CrossRef] [PubMed] 
13. Torraca, V.; Masud, S.; Spaink, H.P.; Meijer, A.H. Macrophage-pathogen interactions in infectious diseases: New therapeutic insights from the zebrafish host model. Dis. Model. Mech. 2014, 7, 785-797. [CrossRef] [PubMed]

14. Gomes, M.C.; Mostowy, S. The Case for Modeling Human Infection in Zebrafish. Trends Microbiol. 2020, 28, 10-18. [CrossRef] [PubMed]

15. Hernãndez, Y.L.; Yero, D.; Pinos-Rodrãguez, J.M.; Gibert, I. Animals devoid of pulmonary system as infection models in the study of lung bacterial pathogens. Front. Microbiol. 2015, 6, 38. [CrossRef]

16. Masud, S.; Torraca, V.; Meijer, A.H. Modeling Infectious Diseases in the Context of a Developing Immune System. Curr. Top Dev. Biol. 2017, 124, 277-329. [CrossRef] [PubMed]

17. Rennekamp, A.J.; Peterson, R.T. 15 years of zebrafish chemical screening. Curr. Opin. Chem. Biol. 2015, 24, 58-70. [CrossRef] [PubMed]

18. Zon, L.I.; Peterson, R.T. In vivo drug discovery in the zebrafish. Nat. Rev. Drug Discov. 2005, 4, 35-44. [CrossRef]

19. Eimon, P.M.; Rubinstein, A.L. The use of in vivo zebrafish assays in drug toxicity screening. Expert Opin. Drug Metab. Toxicol. 2009, 5, 393-401. [CrossRef]

20. Terriente, J.; Pujades, C. Use of Zebrafish Embryos for Small Molecule Screening Related to Cancer. Dev. Dyn. 2013, 242, 97-107. [CrossRef]

21. Bernut, A.; Le Moigne, V.; Lesne, T.; Lutfalla, G.; Herrmann, J.-L.; Kremer, L. In Vivo Assessment of Drug Efficacy against Mycobacterium abscessus Using the Embryonic Zebrafish Test System. Antimicrob. Agents Chemother. 2014, 58, 4054-4063. [CrossRef]

22. Brannon, M.K.; Davis, J.M.; Mathias, J.R.; Hall, C.J.; Emerson, J.C.; Crosier, P.S.; Huttenlocher, A.; Ramakrishnan, L.; Moskowitz, S.M. Pseudomonas aeruginosaType III secretion system interacts with phagocytes to modulate systemic infection of zebrafish embryos. Cell. Microbiol. 2009, 11, 755-768. [CrossRef]

23. Clatworthy, A.E.; Lee, J.S.-W.; Leibman, M.; Kostun, Z.; Davidson, A.J.; Hung, D.T. Pseudomonas aeruginosa Infection of Zebrafish Involves both Host and Pathogen Determinants. Infect. Immun. 2009, 77, 1293-1303. [CrossRef]

24. Llamas, M.A.; Van der Sar, A.M. Assessing Pseudomonas Virulence with Nonmammalian Host: Zebrafish. Pseudomonas Methods Protoc. 2014, 1149, 709-721. [CrossRef]

25. Belon, C.; Soscia, C.; Bernut, A.; Laubier, A.; Bleves, S.; Blanc-Potard, A.-B. A Macrophage Subversion Factor Is Shared by Intracellular and Extracellular Pathogens. PLOS Pathog. 2015, 11, e1004969. [CrossRef]

26. Díaz-Pascual, F.; Ortíz-Severín, J.; Varas, M.A.; Allende, M.L.; Chávez, F.P. In vivo Host-Pathogen Interaction as Revealed by Global Proteomic Profiling of Zebrafish Larvae. Front. Cell. Infect. Microbiol. 2017, 7, 334. [CrossRef]

27. Van Soest, J.J.; Stockhammer, O.W.; Ordas, A.; Bloemberg, G.V.; Spaink, H.P.; Meijer, A.H. Comparison of static immersion and intravenous injection systems for exposure of zebrafish embryos to the natural pathogen Edwardsiella tarda. BMC Immunol. 2011, 12, 58. [CrossRef]

28. Kimmel, C.B.; Ballard, W.W.; Kimmel, S.R.; Ullmann, B.; Schilling, T.F. Stages of embryonic development of the zebrafish. Dev. Dyn. 1995, 203, 253-310. [CrossRef]

29. Saraceni, P.R.; Romero, A.; Figueras, A.; Novoa, B. Establishment of Infection Models in Zebrafish Larvae (Danio rerio) to Study the Pathogenesis of Aeromonas hydrophila. Front. Microbiol. 2016, 7, 1219. [CrossRef]

30. Chevalier, S.; Bouffartigues, E.; Bodilis, J.; Maillot, O.; Lesouhaitier, O.; Feuilloley, M.G.J.; Orange, N.; Dufour, A.; Cornelis, P. Structure, function and regulation of Pseudomonas aeruginosa porins. FEMS Microbiol. Rev. 2017, 41, 698-722. [CrossRef]

31. Cassin, E.K.; Tseng, B.S. Pushing beyond the Envelope: The Potential Roles of OprF in Pseudomonas aeruginosa Biofilm Formation and Pathogenicity. J. Bacteriol. 2019, 201, 00050-19. [CrossRef]

32. Moussouni, M.; Berry, L.; Sipka, T.; Nguyen-Chi, M.; Blanc-Potard, A.-B. Pseudomonas aeruginosa OprF plays a role in resistance to macrophage clearance during acute infection. Sci. Rep. 2021, 11, 1-11. [CrossRef] [PubMed]

33. Brazas, M.D.; Hancock, R.E.W. Ciprofloxacin Induction of a Susceptibility Determinant in Pseudomonas aeruginosa. Antimicrob. Agents Chemother. 2005, 49, 3222-3227. [CrossRef]

34. MacRae, C.A.; Peterson, R.T. Zebrafish-based small molecule discovery. Chem. Biol. 2003, 10, 901-908. [CrossRef]

35. Cafora, M.; Deflorian, G.; Forti, F.; Ferrari, L.; Binelli, G.; Briani, F.; Ghisotti, D.; Pistocchi, A. Phage therapy against Pseudomonas aeruginosa infections in a cystic fibrosis zebrafish model. Sci. Rep. 2019, 9, 1527. [CrossRef] [PubMed]

36. Keightley, M.-C.; Wang, C.-H.; Pazhakh, V.; Lieschke, G.J. Delineating the roles of neutrophils and macrophages in zebrafish regeneration models. Int. J. Biochem. Cell Biol. 2014, 56, 92-106. [CrossRef]

37. Mateus, R.; Pereira, T.; Sousa, S.; De Lima, J.E.; Pascoal, S.; Saúde, L.; Jacinto, A. In Vivo Cell and Tissue Dynamics Underlying Zebrafish Fin Fold Regeneration. PLoS ONE 2012, 7, e51766. [CrossRef]

38. Garai, P.; Berry, L.; Moussouni, M.; Bleves, S.; Blanc-Potard, A.-B. Killing from the inside: Intracellular role of T3SS in the fate of Pseudomonas aeruginosa within macrophages revealed by mgtC and oprF mutants. PLoS Pathog. 2019, 15, e1007812. [CrossRef]

39. Chhabra, S.R.; Harty, C.; Hooi, D.S.W.; Daykin, M.; Williams, P.; Telford, G.; Pritchard, D.I.; Bycroft, B.W. Synthetic Analogues of the Bacterial Signal (Quorum Sensing) MoleculeN-(3-Oxododecanoyl)-1-homoserine Lactone as Immune Modulators. J. Med. Chem. 2003, 46, 97-104. [CrossRef] 